\title{
Reliability of Rapid Diagnostic Test (RDT) for Malaria among Pregnant Women in Ido-Ekiti, Western Nigeria
}

\author{
Ibrahim A. O. ${ }^{1}$, Bello I. S. ${ }^{2}$, Shabi O. M. ${ }^{3}$, Gabriel-Alayode E.M. ${ }^{3}$, Agboola S. M. ${ }^{3}$ and Elegbede O. T. ${ }^{3}$ \\ 1. Department of Family Medicine, Federal Teaching Hospital, Ido-Ekiti 371002, Nigeria \\ 2. Department of Family Medicine, Obafemi Awolowo University Teaching Hospital, Ile Ife 220282, Nigeria \\ 3. Department of Family Medicine, Afe Babalola University, Ado-Ekiti 360001, Nigeria
}

\begin{abstract}
Background: Malaria is the commonest cause of anemia in pregnancy with severe consequence on the developing fetus. Recent clamor by the WHO for testing before treatment has driven a need to assess the effectiveness of available Rapid Diagnostic Tests (RDTs). Objective: To determine the reliability of Rapid Diagnostic Tests (RDTs) for malaria among pregnant women booking in Federal Teaching Hospital, Ido-Ekiti, Nigeria. Materials and Methods: A cross-sectional study of 240 pregnant women were recruited at booking over a period of twenty weeks. A semi-structured questionnaire sought information about socio demographic data. Blood samples were taken for malaria and diagnosis was done using RDT and Microscopy. Results: Prevalence of malaria was $22.1 \%$ by microscopy and $27.5 \%$ by RDT. The sensitivity of RDT was $88.7 \%$ and specificity was $89.8 \%$. The positive predictive value (PPV) of RDT was $71.2 \%$, the Negative predictive value (NPV) of RDT was $96.6 \%$ and accuracy was $89.6 \%$. The reliability of RDT was good (Kappa statistics $=0.731$ ). The reliability of RDTs when compared with that of Microscopy was also good (Kappa statistics $=0.722)$. Conclusions: RDTs was an effective screening test for malaria among the respondents. Hence, primary care physician should utilize this resource by advocating for its availability.
\end{abstract}

Key words: Reliability, RDT, malaria, pregnant women.

\section{Introduction}

Malaria parasitic infection transmitted by mosquitoes, is a major public health problem in the tropics [1]. It is one of the devastating infectious diseases, killing more than 1 million people annually [1]. Around 125 million pregnant women live in malaria-endemic areas [2].

According to World Health Organization (WHO), malaria in pregnancy contributes to significant prenatal morbidity and mortality, accounted for 10,000 maternal and 200,000 neonatal deaths per year [3]. The prevalence of malaria in pregnancy is ranged from $19.7 \%$ to $72 \%$ in Nigeria, West Africa [4]. In Nigeria, the comparative prevalence of malaria in pregnancy are; 65.7\% (Anambra); 29\% (Abakaliki); 20\% (Edo); and

Corresponding author: Bello Ibrahim Sebutu, MBBS, MPH (Liverpool), FMCGP, research fields: malaria, health education, sickle cell disease, hypertension and diabetes.
7.7\% (Lagos). The principal impact of malaria infection in pregnancy is due to the presence of parasites in the placenta causing maternal anemia and low birth weight (LBW) [5]. Early and accurate diagnosis and treatment of malaria in pregnancy are key aspects of preventing complications associated with malaria in pregnancy [6]. Malaria diagnosis by physician particularly in remote areas lacking laboratory support; frequently relies on the patients' symptoms [7]. Misdiagnosis often leads to the unnecessary prescription of malaria medications which are becoming increasingly expensive as drug resistance grows globally [7]. Microscopy is the gold standard in malaria diagnosis [8].

Rapid Diagnostic Tests (RDT) for malaria offer the potential to extend accurate malaria diagnosis to areas where microscopy services are not available such as remote locations or after regular laboratory hours [9].

Malaria RDT rely on detection of parasite specific 
antigens proteins circulating in the blood stream [10]. The most common antigens for RDTs are $\mathrm{P}$. falciparum histidine-rich protein-2 (PfHRP-2) and Plasmodium spp lactose dehydrogenase (pLDH) [11].

Test based on PfHRP-2 antigens are specific to P. falciparum, the most dangerous species of malaria and are more readily available and less expensive. When used appropriately, RDT are easy to use and have adequate sensitivity in detecting even submicroscopic infections. The accuracy and reliability of RDT have been evaluated in diagnosing malaria in pregnancy and are compared to microscopy [12].

The reliability (repeatability) of RDT means that the result of the test is replicated if repeated [13]. Cohen in 1960 proposed the Kappa ( $k$ ) statistic to calculate the reliability of a test [13]. Kappa quantifies the extent to which the observed agreement that the observers achieved exceeds that would be expected by chance alone [13]. It is expressed as the proportion of the maximum improvement that could occur beyond the agreement expected by chance alone. The Kappa statistic can be defined by the equation.

Kappa $[13]=\frac{\begin{array}{l}\text { percent agreement } \\ \text { observed }\end{array}}{\begin{array}{c}- \text { percent agreement expected } \\ \text { by chance alone }\end{array}}$
$100 \%$
$\begin{gathered}\text { - percent agreement } \\ \text { expected by chance } \\ \text { alone }\}\end{gathered}$

Landis and Koch suggested that a Kappa greater than 0.75 represents excellent agreement beyond chance, a Kappa below 0.40 represents poor agreement, and a Kappa of 0.40 to 0.75 represents intermediate agreement to good agreement [13].

\section{Materials and Methods}

The study was conducted at the Igogo Ekiti Outreach Centre of the Federal Teaching Hospital, Ido-Ekiti, Ekiti State. Igogo Ekiti is in Moba Local Government Area and about $50.5 \mathrm{~km}$ from the capital city of Ekiti State, Ado-Ekiti [14].

The people of the Local Government Area were predominantly farmers, but women also engage in trading and other entrepreneurial activities. The town has a rural population that are clustered together to form compounds where family members lives [14].

The outreach hospital was set up initially for immunization, nutritional rehabilitation, preventive care, antenatal centre and collection point of insecticide treated bed net (ITNs) for people in its catchment areas. It was later accredited as a centre for rural posting in family medicine. Federal Teaching Hospital (FTH), Ido-Ekiti is located in Ido-Osi Local Government of Ekiti State. FTH is affiliated to Afe Babalola University, Ado - Ekiti. It is an accredited centre for training for both National Postgraduate Medical College of Nigeria and West African College of Physicians in Family Medicine. It has the capacity of 180 beds, and provides specialist care in obstetrics, \& gynecology, surgery, psychiatrics, pediatrics, Internal Medicine, Family Medicine and Community Medicine. It serves as the main referral centre for all the general and private hospitals scattered across the 16 Local Government Areas (LGA's) of the state.

\subsection{Sample Size Estimation}

This was determined using the formula[15] $\mathrm{n}=\mathrm{z}^{2} \mathrm{pq} / \mathrm{d}^{2}$ and $\mathrm{nf}=\mathrm{n} /\{1+\mathrm{n} / \mathrm{N}\}$ where $\mathrm{n}$ is the minimum sample size when population is less than $10,000, \mathrm{~N}$ is estimate of population size, $\mathrm{Z}$ is standard normal deviate set at 1.96 which corresponds to $95 \%$ confidence interval, $d$ is desired level of precision set at 0.05 level, $\mathrm{p}$ is the estimated proportion with the attribute of interest which is $20 \%$ [16]. The minimum sample size was 219. However, in order to allow for unexpected data losses and drop-outs during recruitment, an attrition rate of $10 \%$ was added and a sample size of 240 was used for the study.

\subsection{Participants/Subjects}

The study populations were pregnant women who presented for booking at Igogo outreach branch of the Federal Teaching Hospital Ido-Ekiti. Booked pregnant women were chosen in this study because the sample frame which was used in calculating the sampling 
interval from the available record of the clinic was that of booked pregnant women. Besides, majority of the unbooked pregnant women presented either in labor or with complications and thereby making it difficult to administer the questionnaire onto them. The inclusion criteria were pregnant women who were on first visit during the current pregnancy and consented to the study. The exclusion criteria were pregnancy related complication(s) patient and those who were on treatment for malaria or have just completed anti-malaria within the previous two weeks.

Systematic random sampling was used to select the respondents. The available data at the booking clinic of the outreach branch showed that an average of 40 pregnant women booked weekly in the previous one year, and 800 estimated respondents in twenty weeks of data collection. The sampling interval was 3 , and every fourth consenting booked pregnant woman was recruited into the study after randomly selecting the first participants on each booking day. This was repeated every booking day until the sample size was met.

\subsection{Data Collection}

The respondents were interviewed with a semi-structured questionnaire that sought information about their socio-demographic and obstetric history. The questionnaire which was pre-tested before use was written in English Language. It was translated into Yoruba and back translated into English language by two separate and competent interpreters. Information on the socio-demographic and obstetric history of the respondents was obtained. These quick general and abdominal examinations were done.

Sample collections were carried out on each respondent. Informed consent of each respondent was obtained prior to administration of questionnaire and examination.

\subsection{Sample Collection}

About $3 \mathrm{ml}$ of venous blood sample was collected from each subject using sterile needle and syringe after consent has been sought and given. The collected blood sample was transferred into an anti-coagulated EDTA bottle. The blood was mixed with the anti-coagulant in the sample bottle. The bottle was labeled to match the subject's identity number and the number on the questionnaire by the researcher before being sent to the laboratory.

\subsection{Malaria RDT (Paracheck Pf Device)}

A paracheck $\mathrm{pf} \circledast$ is a form of RDT that was used in this study. All tests were performed following the manufacturer's instructions. The laboratory and clinical personnel were trained to perform and interpret the RDT' result as follows [10]:

The RDT' packet was opened, and the test kit was removed. The respondent's number was written on the test kit. A drop of blood was collected from the EDTA bottle and introduced into the small round role of the test kit using the pipette of the device. About 5 drops of buffer was added into the large hole. After 15 minutes, the result was read as follows.

Positive results means one red line appeared near the letter ' $c$ ' and one red line appeared near the letter ' $t$ '. Negative results means one red line appeared near the letter ' $c$ ' and no red line appeared near the letter ' $t$ '. Invalid results means no control line appeared in window 'c.' If no line appeared near the letter 'c', the test was repeated using a new unopened test packet. The test was performed by 2 independent trained health workers who were blinded to RDT result of each other. The sensitivity, specificity, positive predictive value, negative predictive value and accuracy/reliability of RDT in diagnosing malaria in pregnancy was determined and compared with microscopy as gold standard [10].

\subsection{Microscopic Examination}

Two blood films, one thin and one thick was made from the blood sample for malaria parasitemia. The thick and thin smears were prepared on clean, dry 
microscope glass slides and were allowed to dry. The thin smears were fixed in methanol and both smears stained with $2 \%$ Giemsa BDH laboratory supplies, Poole BH 15ITD England [17]. The slide was then viewed under a microscope using oil immersion at 100x magnification. Staining of the slide and the parasite counting was done by two independent Medical Laboratory Scientists working in the microbiology department who were blinded to the result of the RDT. Initially, the film was examined for the presence of parasite. The presence of one parasite per 100 high power fields was considered a positive test. Two hundred power fields were examined before a slide was considered negative.

The parasite densities of the positive films were determined by counting the number of parasites in relation to the standard number of leukocytes per microliters. If fewer than 10 parasites are counted, more leukocytes were counted up to 500 and the result was recorded as the number of parasites per 500 leukocytes. Parasite density was then calculated using the formula: number of parasites over number of leukocytes multiply by 8000 to give the number of leukocytes of parasites per microliters of blood [11].

In case there was discordance in the results from the two scientists, a senior scientist was recruited to re-examine the slide for approval. Quality control was ensured by the senior scientist who ensured that the authorized standard operating procedure for thin and thick blood films microscopy was strictly followed.

The sensitivity, specificity, positive predictive value, negative predictive value and accuracy was calculated and used as gold standard for the result of Rapid Diagnostic Test $[10,11]$.

\subsection{Data Analysis}

All data collected were analyzed using the statistical package for social sciences (SSPS) for Windows Software Version 16.0.

The socio-demographic characteristics of the respondents were described using appropriate tables.
Chi-Square analysis was used to determine significant of association. Mean value was compared by student-t-tests. The chi-square test and the fisher's exact test were used to compare proportion of categorical variables. A "p" value of $<0.05$ was considered significant.

\subsection{Ethical Clearance and Consent}

Ethical clearance was obtained from the ethical review and research committee of Federal Teaching Hospital, Ido-Ekiti. Informed verbal and written consent were obtained from willing participants. Respondents confidentiality was assured.

\section{Results}

Two hundred and forty women were recruited for the study. The mean age of the respondents (table 1) was $29.1 \pm 5$ years and their ages ranged from 18 years and 44years. Fifty-three (22.1\%) were positive for malaria (Figure 1) by microscopy and $66(27.5 \%)$ by RDT. Therefore, the prevalence of malaria by microscopy was $22.1 \%$ and by RDT was $27.5 \%$. The relationship between malaria parasitemia (Table 1) by age group showed no statistically significant difference $\left(\mathrm{X}^{2}=1.667, \mathrm{P}=0.644\right)$. Also, there was no statistical significant difference by place of domicile $\left(\mathrm{X}^{2}=0.332\right.$, $\mathrm{P}=0.565)$, occupation $\left(\mathrm{X}^{2}=2.121, \mathrm{P}=0.311\right)$, respondent education $\left(X^{2}=3.166, P=0.367\right)$, family type $\left(\mathrm{X}^{2}=0.249, \mathrm{P}=0.617\right)$, family size $\left(\mathrm{X}^{2}=0.215, \mathrm{P}\right.$ $=0.898)$, monthly income $\left(X^{2}=1.933, P=0.380\right)$, Socio-economic status $\left(\mathrm{X}^{2}=0.067, \mathrm{P}=0.796\right)$, parity $\left(\mathrm{X}^{2}=2.703, \mathrm{P}=0.100\right)$, and Gestational age $\left(\mathrm{X}^{2}=\right.$ 5.297, $\mathrm{P}=0.071)$. very few $29(12.1 \%)$ of the respondents with malaria parasitemia (table 2) using microscopy had their parasite density ranged between $1,001-5,000$ while nearly half $25(53.2 \%)$ who were positive with RDT (table 3 ) had their parasite density ranged between $1001-5000$. On the whole, whether microscopy or RDT was used to diagnose malaria among the respondents, there seems no statistically significance differences in the result obtained. 
Table 1 Socio demographic data of the respondents and distributions of positive malaria infection by RDTs compared with microscopy.

\begin{tabular}{|c|c|c|c|c|c|}
\hline $\begin{array}{l}\text { Positive MP } \\
\text { Characteristic } \\
\text { Microscopy }\end{array}$ & RDT & & $\left(X^{2}\right)$ & df & $p$-value \\
\hline \multicolumn{6}{|l|}{ Age group (yrs) } \\
\hline Less than 25 yrs & $14(26.4)$ & $17(25.8)$ & 1.667 & 3 & 0.644 \\
\hline $25-29$ yrs & $16(30.2)$ & $17(25.8)$ & & & \\
\hline $30-34$ yrs & $18(34.0)$ & $25(37.9)$ & & & \\
\hline 35 yrs and above & $5(9.4)$ & $7(10.6)$ & & & \\
\hline \multicolumn{6}{|l|}{ Domicile } \\
\hline Urban Ekiti & $3(5.7)$ & $5(7.6)$ & 0.332 & 1 & 0.565 \\
\hline Rural Ekiti & $50(94.4)$ & $61(92.4)$ & & & \\
\hline \multicolumn{6}{|c|}{ Respondent's occupation } \\
\hline Civil servants & $1(1.9)$ & $1(1.5)$ & 2.112 & 2 & 0.311 \\
\hline Self employed & $34(64.1)$ & $42(63.9)$ & & & \\
\hline Unemployed & $18(34.0)$ & $23(34.9)$ & & & \\
\hline \multicolumn{6}{|c|}{ Respondent's education } \\
\hline Tertiary & $1(1.9)$ & $1(1.5)$ & 3.166 & 3 & 0.367 \\
\hline Secondary & $5(9.4)$ & $4(6.1)$ & & & \\
\hline Primary & $45(84.9)$ & $59(89.4)$ & & & \\
\hline None & $2(3.8)$ & $2(3.0)$ & & & \\
\hline \multicolumn{6}{|l|}{ Marital status } \\
\hline Single & $0(0.0)$ & $0(0.0)$ & NA & & \\
\hline Married & $53(100.0)$ & $66(100.0)$ & & & \\
\hline \multicolumn{6}{|l|}{ Type of family } \\
\hline Monogamous & $40(75.5)$ & $48(72.7)$ & 0.249 & 1 & 0.617 \\
\hline Polygamous & $13(24.5)$ & $18(27.3)$ & & & \\
\hline \multicolumn{6}{|l|}{ Family size } \\
\hline$<5$ & $30(56.6)$ & $34(51.5)$ & 0.215 & 2 & 0.898 \\
\hline 6-step & $17(32.1)$ & $25(37.9)$ & & & \\
\hline$>10$ & $6(11.3)$ & $7(10.6)$ & & & \\
\hline \multicolumn{6}{|c|}{ Family monthly income } \\
\hline Low & $23(43.4)$ & $25(37.9)$ & 1.933 & 2 & 0.380 \\
\hline Middle & $24(45.3)$ & $30(45.3)$ & & & \\
\hline High & $6(11.3)$ & $11(16.7)$ & & & \\
\hline \multicolumn{6}{|l|}{ Socio-economic status } \\
\hline High & $0(0.0)$ & $0(0.0)$ & 0.067 & 1 & 0.796 \\
\hline Middle & $4(7.5)$ & $6(9.1)$ & & & \\
\hline Low & $49(92.5)$ & $60(90.9)$ & & & \\
\hline \multicolumn{6}{|l|}{ Parity } \\
\hline Primigravidae & $23(43.5)$ & $24(36.4)$ & 2.703 & 1 & 0.100 \\
\hline Multigravidae (2-5) & $30(56.6)$ & $42(63.6)$ & & & \\
\hline \multicolumn{6}{|l|}{ GA (trimester) } \\
\hline First trimester & $11(20.8)$ & $15(22.7)$ & 5.297 & 2 & 0.071 \\
\hline Second trimester & $39(73.6)$ & $45(68.2)$ & & & \\
\hline Third trimester & $3(5.7)$ & $6(9.1)$ & & & \\
\hline
\end{tabular}




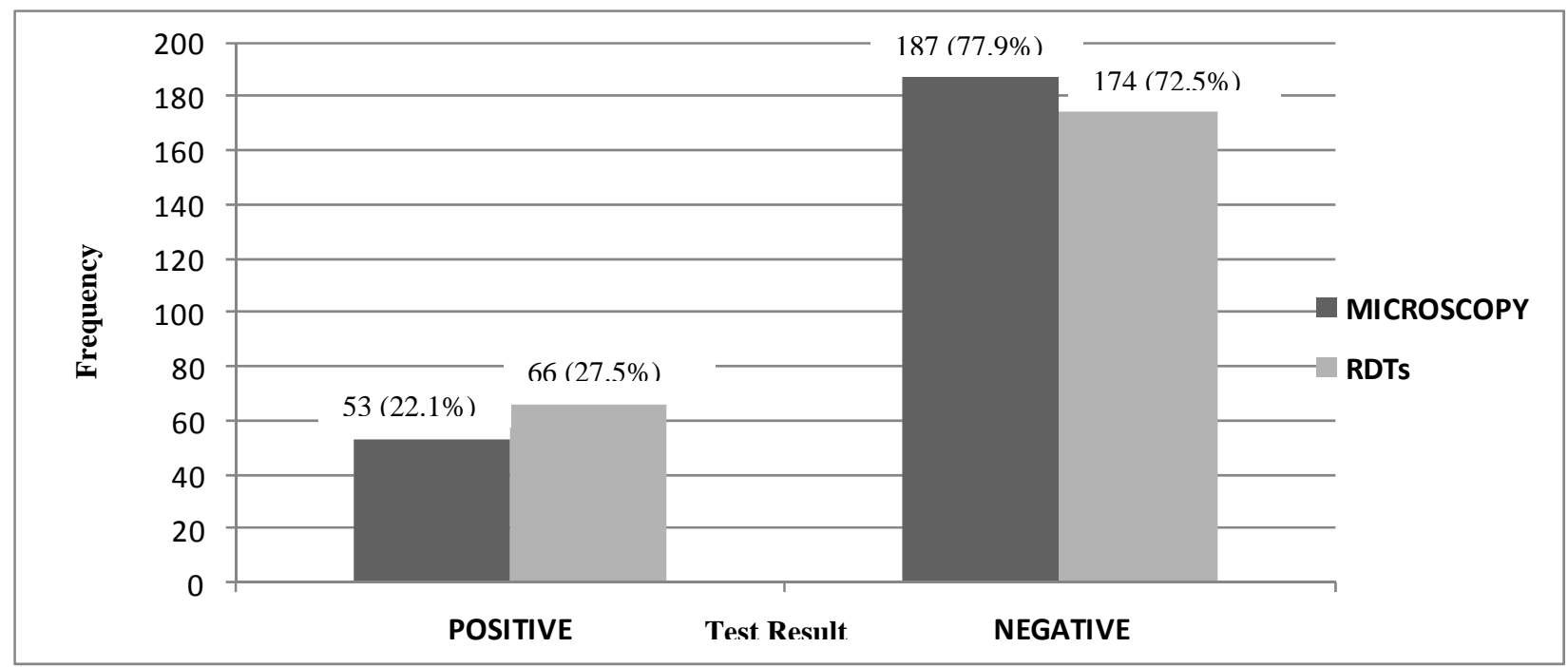

Fig. 1 Results obtained from RDT and microscopy.

Table 2 Malaria parasite density by microscopy among the study respondents.

\begin{tabular}{lll}
\hline Parasite density $($ per $\mu \mathrm{L})$ & Frequency & Percent \\
\hline 0 & 187 & 77.9 \\
$1-500$ & 6 & 2.5 \\
$501-1,000$ & 14 & 5.8 \\
$1,001-5,000$ & 29 & 12.1 \\
$>5,000$ & 4 & 1.7 \\
Total & 240 & 100.0 \\
\hline
\end{tabular}

NB: Mean parasite density $=2,197.7$ with a range of $400-7,160$ per $\mu \mathrm{L}$.

Table 3 RDT result distribution by parasite density categories.

\begin{tabular}{|c|c|c|c|c|c|}
\hline RDT & & \multicolumn{3}{|c|}{ Parasite density per $\mu \mathrm{L}$} & Total \\
\hline Result & $<500$ & $501-1,000$ & $1,001-5,000$ & $>5,000$ & \\
\hline Positive & $6(12.8)$ & $12(25.5)$ & $25(53.2)$ & $4(8.5)$ & $47(100.0)$ \\
\hline Negative & $0(0.0)$ & $2(33.3)$ & $4(66.7)$ & $0(0.0)$ & $6(100.0)$ \\
\hline Total & $6(11.3)$ & $14(26.4)$ & $29(54.7)$ & $4(7.5)$ & $53(100.0)$ \\
\hline
\end{tabular}

Nearly half of the respondent with a positive RDT result had parasite densities ranging between 1,001-5,000 per $\mu \mathrm{L}$.

In this study, the sensitivity of RDT (Table 4) was found to be $88.7 \%$, the specificity was $89.8 \%$, its Positive Predictive Value (PPV) was 71.2\%. Negative Predictive Value (NPV) was $96.6 \%$ and its accuracy was 89.6\%. the reliability of RDT (Table 5) was calculated using the Kappa Statistics. 27.5\% (66/240) of the subjects identified as positive by observer 1 using RDT. If this has been by chance alone, then only $27.5 \%$ of the subjects identify as positive by observer $2(n=68)$ were expected to have been identified as such by observer $1(27.5 \%$ of $68=18.7)$. Similarly, $72.5 \%(174 / 240)$ of the subjects were identified as Negative by observer 1 $(72.5 \%$ of $172=124.7)$. The Kappa Statistic was therefore calculated as 0.731 . Also, the reliability of RDT (Table 6) was compared with that of Microscopy. $22.1 \%(53 / 240)$ of the subjects were identified as positive on Microscopy. If this had been by chance alone, then only $22.1 \%$ of the subjects identified as positive by RDT ( $n=66$ ) were expected to have been identified as such by Microscopy ( $22.1 \%$ of $66=14.6$ ). Similarly, $77.9 \%(187 / 240)$ of the subjects identified as negative on Microscopy and if this had been by chance also, then only $77.9 \%$ of the subjects identified as negative RDT $(n=174)$ were expected to have been identified as negative by Microscopy ( $77.9 \%$ of $174=135.6$ ). The Kappa statistic was therefore calculated as 0.722 . 
Table 4 Sensitivity, specificity, predictive values and accuracy of RDT.

\begin{tabular}{llll}
\hline \multirow{2}{*}{ RDTs } & \multicolumn{3}{c}{ Microscopy } \\
\cline { 2 - 4 } & Yes & No & \\
\hline Yes & $47(\mathrm{TP})$ & $19(\mathrm{FP})$ & $66(\mathrm{TP}+\mathrm{FP})$ \\
No & $6(\mathrm{FN})$ & $168(\mathrm{TN})$ & $174(\mathrm{TN}+\mathrm{FN})$ \\
Total & $53(\mathrm{TP}+\mathrm{FN})$ & $187(\mathrm{TN}+\mathrm{FP})$ & 240 \\
\hline
\end{tabular}

$\mathrm{TP}=$ True positive; $\mathrm{FP}=$ False positive; $\mathrm{TN}=$ True negative; $\mathrm{FN}=$ False negative.

Sensitivity, specificity, positive/negative predictive values and accuracy of RDT:

Sensitivity $=\frac{\mathrm{TP}}{\mathrm{TP}+\mathrm{FN}} \times 100 \%=88.7 \%$;

Specificity $=\frac{\mathrm{TN}}{\mathrm{TN}+\mathrm{FP}} \times 100 \%=89.8 \%$;

Positive predictive value $(\mathrm{PPV})=\frac{\mathrm{TP}}{\mathrm{TP}+\mathrm{FP}} \times 100 \%=71.2 \%$;

Negative predictive value $(\mathrm{NPV})=\frac{\mathrm{TN}}{\mathrm{TN}+\mathrm{FN}} \times 100 \%=96.6 \%$;

Accuracy $=\frac{\mathrm{TP}+\mathrm{TN}}{\text { Grand Total }} \times 100 \%=89.6 \%$.

Table 5 Reliability of RDT.

\begin{tabular}{llll}
\hline & \multicolumn{2}{c}{ Observer 1 } & Total \\
\cline { 2 - 3 } Observer & Positive & Negative & 68 \\
\hline Observed numbers & 54 & 14 & 172 \\
\hline Positive & 12 & 160 & 240 \\
Negative & 66 & 174 & 68 \\
Total & 18.7 & & 172 \\
\hline Expected numbers & 47.3 & 49.3 & 240 \\
\hline Positive & 66 & 124.7 & \\
Negative & 174 & \\
Total & &
\end{tabular}

$k=$ Kappa statistics $=\frac{A_{\text {obs }}-A_{\exp }}{1-A_{\exp }}=(0.892-0.598) /(1-0.598)=0.294 / 0.402=0.731$.

Table 6 Reliability of RDT compared with Microscopy.

\begin{tabular}{llll}
\hline \multirow{2}{*}{ RDTs } & \multicolumn{2}{c}{ Microscopy } & Total \\
\cline { 2 - 4 } & Positive & Negative & 66 \\
\hline Observed numbers & & & 174 \\
\hline Positive & 47 & 19 & 240 \\
Negative & 6 & 187 & 66 \\
Total & 53 & & 174 \\
\hline Expected numbers & & 51.4 & 240 \\
\hline Positive & 14.6 & 135.6 & \\
Negative & 38.4 & 187 & \\
Total & 53 & 187 &
\end{tabular}

$k=$ Kappa statistics $=\frac{A_{\text {obs }}-A_{\text {exp }}}{1-A_{\exp }}=(0.896-0.626) /(1-0.626)=0.270 / 0.374=0.722$.

\section{Discussion}

4.1 Reliability of RDT for the Diagnosis of Malaria in Pregnancy

The reliability of RDT in the diagnosis of malaria in pregnancy in the study was established by calculating the level of agreement (Kappa statistic) between the study of RDTs obtained by the two observers who independently performed and read the result of RDTs. The study revealed that Kappa statistic of RDTs was 0.731. Therefore, reliability of RDT in the study was good, this study also revealed that paracheck $\mathrm{pf} \circledast$ 
RDT had a sensitivity of $88.7 \%$, specificity of $89.8 \%$, positive predictive value (PPV) of $71.2 \%$, and Negative Predicative Value (NPV) of 96.0\%.

Using the exact same kits (Paracheck $®$ ) used in this study, Umeh and colleagues in Owerri, Imo state reported the sensitivity of $86.7 \%$, PPV of $93.7 \%$ and NPV of $94.5 \%{ }^{9}$ The result was similar to the findings in this study.

Another study by Muhindo et al in Democratic Republic of Congo using paracheck ${ }^{\circledR}$ RDT found sensitivity of 87.8 and specificity of 91.6\%.[18] This result was also similar to the findings in this study.

A cross sectional study by Bisofi et al. [19] reported sensitivity of $86 \%$ and specificity of $90 \%$, PPV of $84 \%$ and NPV of $78 \%$. This result was less than findings in this study.

Therefore, as screening tests, the result of this study showed a good performance with the RDT kit, when repeated, being able to detect the presence of malaria infection and defined is absence as stated above. Thus, if an application were to be drawn from this study for poor resources settings, it can be argued that the use of RDT would be a favored alternative diagnostic tool because of the beneficial effect of treating more women for malaria within a short time.

\subsection{Reliability of RDT in Comparison with Microscopy for the Diagnosis of Malaria in Pregnancy}

The reliability of RDT in comparison with microscopy in this study was established by calculating the level of agreement (Kappa statistic) of RDT in relation to that of microscopy among pregnant women ages $18-44$ years.

The study revealed that Kappa statistic was 0.722, therefore, reliability of RDT when compared with microscopy was good. The false negative of 6 (11.3\%) in this study may be due to low parasitemia. These agree with similar studies with other RDTs where false negative results were reported in patient with parasitemia of less than 50 parasites of blood. [20] Furthermore, some studies have reported that false negative report with RDT could be as a result of mutant parasite that escape the antigenic determinant of the RDT.[20]

The false positive in this study is 19 (35.8\%). The reason for this (35.8\%) could possibly be explained by the fact that patients who had treated and whose parasitemia has cleared could still be have tested positive by the RDT picking up ruminant antigens.[21 ]Studies shown antigens to be present several days after clearance of parasitemia from the blood stream making the RDTs unsuitable for monitoring treatment and level of parasitemia.[21] Also, the sequestration of parasitized erythrocytes in tissue capillaries and placental malaria in the absence of peripheral blood parasitemia may have also yielded positive RDT and negative microscopy for malaria parasites.

The specificity of RDT kit used in the study was 89.8\%. This showed that in nearly all the tests subjects, RDT can define or detect the absence of $P$. falciparum as evidenced by lack of antibodies to PF HRP-2. Also, the PPV in this study was $71.2 \%$. This means treatment of these positive women with anti-malarias might provide some prophylactic effect against subsequent infection outweighing the risk of not treating genuine outcome. The NPV in this study was $96.6 \%$. This is good at reassuring that the respondents does not have malaria infection and correctly identifies $89.9 \%$ of those who do not have malaria infection (the specificity).

Also, at the time of this study, a single pack of 25 RDTs unit was sold by commercial distributor at a cost of Six Thousand Naira $(6,000.00)$ or Two Hundred and forty Naira (\$1.50) per kit. On the other hand, the hospital fees for Giemsa microscopy is five hundred naira (N500.00 or \$1.43 equivalent) for adult patients. The apparent lower cost of RDT, would be beneficial and encourage a wide-spread use in resource constrained malaria endemic countries. This will enhance rapid malaria case diagnosis and management, and play an important role in the fight 
against malaria. Besides, certain challenges arise in providing the facility for accurate microscopic diagnosis of malaria at the rural communities. This is the setting where the malaria menace threatens the life of the pregnant women and the unborn babies. It can strongly be asserted that the findings of this study were good to suggest the adoption of the RDT in the parasitological confirmation of malaria diagnosis. As stated by WHO, this will, with some degree of certainty, help to rule out malaria in pregnant women.[8 ]

\section{Conclusions}

This study has shown that there was no significant differences in using RDT or microscopy in the diagnosis of malaria in pregnancy. It implied that RDT could be used in place of microscopy especially in poor resource setting like this study area. Future researchers should focus on large studies involving a community based general population of women so that better evaluation of the reliability of the RDT can be made and compared with the microscopy.

\section{Limitation}

This study covered only pregnant women who presented themselves for booking. There is need to conduct similar research on non pregnant women to show if there is difference in the sensitivity and specifity of RDTs between the two groups.

Declaration of interest: The authours declare no interest.

\section{References}

[1] Schantz-Dunn, J., and Nour, N. M. 2009. "Malaria and Pregnancy: A Global Health Perspective." Rev Obstet Gynecol 2 (3): 186-92.

[2] Raimi, O. G., and Kanu, C. P. 2010. "The Prevalence of Malaria Infection in Women Living in a Suburb of Lagos, Nigeria." African Journal of Biochemistry Research 4 (10): 243-5.

[3] World Health Organization (WHO). 2012. World Malaria Report. Switzerland: WHO.

[4] Agomo, C. O., and Oyibo, W. A. 2013. "Factors Associated with the Risk of Malaria Infection among
Pregnant Women in Lagos, Nigeria." Infectious Disease of Poverty 2 (1): 19.

[5] Okofor, I. M., Mbah, M., and Usanga, E. A. 2012. "The Impact of Anaemia and Malaria Parasite Infection in Pregnant Women. Nigeria Perspective." IOSR Journal of Dental and Medical Sciences 1 (1): 34-8.

[6] Chukwuocha, U. M., Dozie, I. N., and Chukwuocha, A. N. 2012. "Malaria and Its Burden among Pregnant Women in Parts of the Niger Delta Area of Nigeria." Asian Pacific Journal of Reproduction 1 (2): 147-51.

[7] World Health Organization (WHO). 2010. Guidelines for the Treatment of Malaria. Geneva, Switzerland: WHO.

[8] World Health Organization (WHO). 2012. "Results of: WHO Product Testing of Malaria Rapid Diagnostic Tests." In Malaria Rapid Diagnostic Test Performance. Round 1 (2008). Geneva: WHO.

[9] Uneh, S. I., Enwuru, C. P., and Egbuobi, R. C. 2013. "Diagnosis of Malaria Pregnancy: A Comparison of Microscopy with Rapid Diagnostic Tests." Net Journals of Microbiology Research International 1 (3): 35-9.

[10] Minja, D. T., Schmiegelow, C., Oesterholt, M., Magistrado, P. A., Bostrum, S., John, D., et al. 2012. "Reliability of Rapid Diagnostic Tests in Diagnosing Pregnancy Associated Malaria in North-Eastern Tanzania." Malar J. 11 (1): 211.

[11] Major, A., Moro, L., Aguilar, R., Bardají, A., Cisteró, P., Serra-Casas, E., Sigaúque, B., Alonso, P. L., Ordi, J., and Menéndez, C. 2012. "How Hidden Can Malaria Be in Pregnant Women? Diagnosis by Microscopy, Placental History, Polymerase Chain Reaction and Detection of Histamine-Rich Protein 2 in Plasma." Clin Infect Dis. 54 (11): 1561-8.

[12] Batwala, V., Magnussen, P., and Nuwaha, F. 2010. "Are Rapid Diagnostic Tests Accurate in Diagnosis of Plasmodium Falciparum Malaria Compared to Microscopy at Rural Health Centers?" Malar J 9: 349.

[13] Gordis, L. 2009. Textbook of Epidemiology. 4th edit. Philadelphia: Saunders Elsevier, 85-108.

[14] Amase, N., Dayo, E. L., Helen, E. A., and Aliu, A. A. 2014. "Malaria Parasitaemia among Pregnant Women Possessing Freely Donated Insecticide-Treated Nets (ITNs) in Ado-Ekiti, Nigeria." Global Journal of Biology, Agriculture and Health Sciences 3 (1): 86-91.

[15] Araoye, M. O. 2003. "Subject Selections: Sample Size Determination." In Research Methodology with Statistics for Health and Socio Statistics, edited by Araoye, M. O. Ilorin: Nathadex Publishers, 117-8.

[16] Enato, E. F. O., Men, P. F., Klhamafe, A. O., Okpere, E. E., Pogoson, E., and Schallig, H. D. F. 2009. "Plasmodium Falciparum Malaria in Pregnancy: Prevalence of Peripheral Parasitaemia Malaria Care-Seeking Behavour among Pregnant Women 
Attending Two Antenatal Clinic in Edo State Nigeria." Journal of Obstetrics and Gynaecology 29 (4): 301-6.

[17] Okonko, I. O., Soloye, F. A., Amusan, T. A., Ogun, A. A., Udeze, A. O., Nakang, A. O., et al. 2009. "Prevalence of Malaria Plasmodium in Abeokuta, Nigeria." Malaysian Journal of Microbiology 5 (2): 113-8.

[18] Muhindio, H. M., Ilombe, G., Meye, R., Mitashi, P. M., Kutekemeni, A., Gasigwa, D., Lutumba, P., and Van Geertruyden, J. P. 2012. "Accuracy of Malaria Rapid Diagnostic Test Optimal-IT ${ }^{(\mathrm{R})}$ in Kinshasa, the Democratic Republic of Congo.” Malar J. 11: 224.

[19] Biosffi, Z., Sirima, S. B., Menten, J., Pattaro, C., Angheben, A., Gobbi, F., Tinto, H., et al. 2010. “Accuracy of a Rapid Diagnostic Test on the Diagnosis of Malaria
Infection and Malaria-Attributable Fever during Low and High Transmission Season in Burkina Faso." Malar $J$ 9: 192.

[20] Mayxay, M., Pukrittayakamee, S., Chotivanich, K., Looareesuwan, S., and White, N. J. 2001. "Persistence of Plasmodium Falciparum HRP-2 in Successfully Treated Acute Falciparum Malaria." Trans R. Soc Trop Med Hyg 95 (2): 179-82.

[21] Dhorda, M., Piola, P., Nyechangane, D., Tumwebaze, B., Nalusji, A., Nabasumba, C., Turyakira, E., et al. 2012. "Performance of a Histidine-Rich Protein 2 Rapid Diagnostic Test, Parachack Pf®, for Detection of Malaria Infection in Uganda Pregnant Women.” Am J Trop Med Hyg 86 (1): 93-5. 